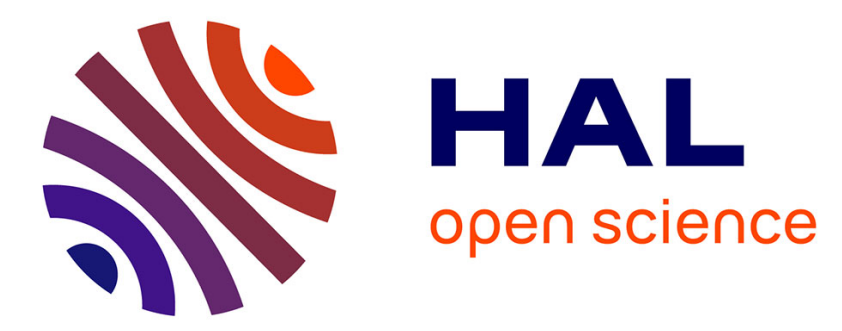

\title{
Droplet breakup in microfluidic T-junctions at small capillary numbers
}

\author{
Marie-Caroline Jullien, M.-J. Tsang Mui Ching, Céline Cohen, Laure \\ Menetrier, Patrick Tabeling
}

\section{- To cite this version:}

Marie-Caroline Jullien, M.-J. Tsang Mui Ching, Céline Cohen, Laure Menetrier, Patrick Tabeling. Droplet breakup in microfluidic T-junctions at small capillary numbers. Physics of Fluids, 2009, 21 (7), pp.072001. 10.1063/1.3170983 . hal-00991895

\section{HAL Id: hal-00991895 https://hal.science/hal-00991895}

Submitted on 16 May 2014

HAL is a multi-disciplinary open access archive for the deposit and dissemination of scientific research documents, whether they are published or not. The documents may come from teaching and research institutions in France or abroad, or from public or private research centers.
L'archive ouverte pluridisciplinaire HAL, est destinée au dépôt et à la diffusion de documents scientifiques de niveau recherche, publiés ou non, émanant des établissements d'enseignement et de recherche français ou étrangers, des laboratoires publics ou privés. 


\title{
Droplet breakup in microfluidic T-junctions at small capillary numbers
}

\author{
M.-C. Jullien, M.-J. Tsang Mui Ching, C. Cohen, L. Menetrier, and P. Tabeling \\ MMN, CNRS, ESPCI Paris-Tech, 10 rue Vauquelin, 75005 Paris, France
}

(Received 4 November 2008; accepted 4 June 2009; published online 17 July 2009)

\begin{abstract}
We perform experimental studies of droplet breakup in microfluidic T-junctions in a range of capillary numbers lying between $4 \times 10^{-4}$ and $2 \times 10^{-1}$ and for two viscosity ratios of the fluids forming the dispersed and continuous phases. The present paper extends the range of capillary numbers explored by previous investigators by two orders of magnitude. We single out two different regimes of breakup. In a first regime, a gap exists between the droplet and the wall before breakup occurs. In this case, the breakup process agrees well with the analytical theory of Leshansky and Pismen [Phys. Fluids 21, 023303 (2009)]. In a second regime, droplets keep obstructing the T-junction before breakup. Using physical arguments, we introduce a critical droplet extension for describing the breakup process in this case. (c) 2009 American Institute of Physics.
\end{abstract}

[DOI: $10.1063 / 1.3170983$ ]

The idea of developing biochemical and chemical processes in microdroplets rather than in microchannels or microchambers was proposed recently. ${ }^{1-3}$ Among the main advantages of the microdroplet approach, one may mention the absence of contact between the chemical species and the walls (although there is still a contact with the droplet boundaries), the possibility of varying, in each droplet, the physicochemical conditions under which chemical or biochemical processes develop (opening the way to screening), and the fast mixing that takes place in each droplet. ${ }^{1-3}$ In the future, one may envision microfluidic systems with mazes of microchannels along which droplets conveying solutes, materials, and particles undergo transformations, reactions, and processes. Such systems could be used to perform all sorts of bio-/physicochemical analysis or to produce novel entities. Such a prospective requires the control of elementary operations such as droplet merging, breakup, transport, exchanges, redirection, and storage. This motivated a number of research groups to develop "digital microfluidics," a topic entirely dedicated to the handling of droplets in microsystems. ${ }^{4}$

From a fluid dynamical viewpoint, digital microfluidics deals with moving interfaces that are known to develop subtle and sometimes amazingly complex dynamics. Complex dynamical behavior of the interfaces obviously hinders droplet control. However, in typical microfluidic situations, capillary numbers are small and therefore viscous tangential stresses that distort the interfaces and generate complexity at vanishingly small Reynolds numbers play a minor role compared to the capillary forces that tend to minimize the interfacial area, and which, combined to the effect of the confinement, favor simple patterns. Small capillary number constraints are systematically exploited by theoretical investigators to provide simplified descriptions of elementary processes such as droplet formation in T-junctions and focusing geometries, or droplet breakup in various geometries. ${ }^{5-8}$

This present paper focuses on one of these elementary processes-droplet breakup in microfluidic junctions. Several experimental, analytical, and numerical studies have been published in literature on this subject. Most of the stud- ies focus on the T-junctions, where a channel splits into two symmetric arms, forming a T. In such systems, depending on droplet size, capillary number and viscosity ratio of the dispersed and continuous phase, droplets arriving at the junction may either breakup or flow along one of the arm of the T., ${ }^{5,7}$ In the description of Ref. 5, the droplets are elongated by the action of velocity gradients up to a point where Rayleigh Plateau conditions are met, giving rise to breakup. The physical description and the scaling law that was obtained in Ref. 5 was supported numerically and experimentally in a range of capillary numbers lying between $2 \times 10^{-3}$ and $7 \times 10^{-2}$ and for a range of viscosity ratios extending from 1 to $7 .{ }^{9}$ In a recent paper, Leshansky and Pismen ${ }^{10}$ developed an analytical theory that describes the breakup process in two-dimensional (2D) T-junctions. They could determine the conditions under which stationary solutions exist. This range corresponds, physically, to nonbreakup conditions. In the present paper, we shall confront in some detail this theory to our observations.

The case of junctions of arbitrary angles considered in Ref. 6 was analyzed in a range of capillary numbers lying between $10^{-6}$ and $10^{-2}$. As compared to Refs. 5 and 10, this work proposed a different view of the breakup process. By exploiting the fact that droplets develop circular fronts and by considering the geometrical constraints they are subjected to, a geometrical description of the breakup process was proposed. This description compared well with experiments using different fluid systems and flow geometries. Although the approaches developed thus far for symmetric and nonsymmetric junctions analyze the same physical phenomenon, they differ conceptually and at the moment no consistent picture that would embrace the two viewpoints has been proposed.

In the present paper, we carry out series of breakup experiments in symmetric T-junctions for a range of capillary numbers extending from $4 \times 10^{-4}$ to $2.5 \times 10^{-1}$ and for two fluid systems. The present work extends the range explored thus far in the literature by two orders of magnitudes. More importantly, it encompasses the capillary number ranges con- 


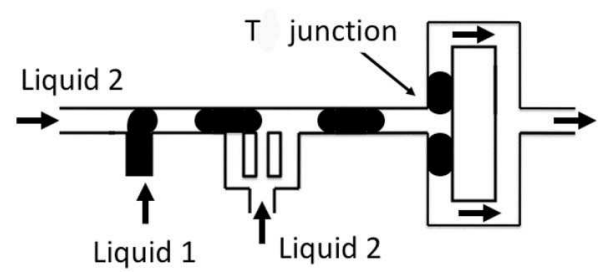

FIG. 1. Sketch of the experiment: water droplets are formed in a microchannel junction and move toward a T-junction where, depending on the flow conditions, they breakup or simply bifurcates into one of the branches of the junction.

sidered in Ref. 5 and 6. In the course of the present work, we discovered a regime that has not been reported yet. In this paper, we will introduce a notion of critical droplet extension to describe such a regime. As a whole, the present work offers for the first time a picture of the breakup process in microfluidic T-junctions for small and moderately small capillary numbers that is consistent with different approaches developed in this area. ${ }^{5,6,10}$

The series of experiments we describe here are sketched in Fig. 1. The microchannel includes a junction in which, depending on the liquids we used, water-in-oil or oil-inwater droplets are formed. Downstream the droplet generator, a series of entries forming a comb injects additional continuous phase, separating the droplets by variable lengths, typically several hundreds of micrometers. Further downstream, the microchannel divides in two parts beyond a first T-junction. The two channels eventually merge at a second node to form a single channel again. To drive the fluids, we use either gravitational forces or pressure sources (MFCS Fluigent) that allow imposing inlet pressures with $1 \%$ accuracy in a range lying between 0 and 1 bar. By injecting the continuous phase through the comb, the distances separating two successive droplets can be increased and the numbers of droplets in the divided-channel section after the T-junction can be varied between one and five. To avoid complications, most of the experiments were carried out with a number equal to one. Throughout the paper, we concentrate our attention at the first T-junction in which, depending on the experimental conditions, droplets may or not breakup.

Concerning microfabrication, the systems are mold in polydimethylsiloxane (PDMS), using soft lithography; throughout the experiments, the microchannels have the following cross-sectional dimensions: $80 \mu \mathrm{m}$ high and $80 \mu \mathrm{m}$ wide. The channel walls are in PDMS. We worked with two fluid systems: for the first one, we used de-ionized (DI) water and hexadecane with $1 \% w / w$ SPAN80, a concentration well above the critical micellar concentration. The interfacial ten- sion between the two fluids is $5 \mathrm{mN} / \mathrm{m}$. In the second fluid system, we used FC 3283 3M fluorinated oil and DI water with $1 \% \mathrm{w} / \mathrm{w}$ sodium dodecyl sulfate (SDS) and an interfacial tension of $15 \mathrm{mN} / \mathrm{m}$. Throughout the experiments, we span a range of flow rates varying between 0 and $40 \mu \mathrm{l} / \mathrm{mn}$. The corresponding capillary numbers $\mathrm{Ca}=\mu U / \gamma$ we investigated (where $U$ is the speed of the droplet arriving at the T-junction, $\mu$ is the external phase viscosity, and $\gamma$ is the oil-water interfacial tension) range between $4 \times 10^{-4}$ and $2.5 \times 10^{-1}$ thus spanning almost three orders of magnitude. Droplet speeds along with various geometrical quantities (droplet size, front location) are determined by image processing. The two viscosity ratios $\lambda=\mu_{d} / \mu_{c}$ that we consider here (where $\mu_{d}$ and $\mu_{c}$ are the viscosities of the dispersed and continuous phases, respectively) are equal to 1.67 and 0.11 for the water/alcane and fluorinated oil/water, respectively. The main characteristics of the experiments are summarized in Table I.

We propose here a classification of the phenomena we observed. It includes a distinction between "obstructed" (or blocked) and "nonobstructed" flows. We consider here that there is obstruction when the droplet, seen from above, touches the walls of the T-junction, i.e., if the distance between the droplet and the wall is below the optical resolution. In the nonobstructed situations a visible gap, or tunnel, takes place between the droplet and the walls of the T-junction. Our definition of obstruction is purely geometrical. It overlooks the possibility that fluid may flow along the corners of the microchannel or between the curved droplet interface and the sidewalls. We shall address its dynamical significance later on. For the moment, we use our definition of obstruction to classify the different types of breakup that are observed in the experiment.

(a) No breakup [Fig. 2(a)]. The droplet, as it arrives at the T-junction (a), reduces its speed, penetrates into the junction (b) and then, under the effect of a random disturbance, bifurcates into either branch of the junction (c). Two subcases can be singled out: In a first case [shown in Fig. 2(a)], the droplet penetrates into the junction keeping the arms obstructed, then opens a tunnel on one side of the junction and eventually moves in the opposite direction. In another case, the droplets move in one of the T-junction arms, leaving a tunnel open throughout the process.

(b) Breakup with tunnels [Fig. 2(b)]. The droplet arrives at the junction and penetrates symmetrically into the two branches of the junction. As for regime A, two subcases can be singled out: In a first case [shown on Fig. 2(b)],

TABLE I. Working liquids and experimental specifications.

\begin{tabular}{lcccc}
\hline \hline Working liquids & $\lambda=\mu_{d} / \mu_{c}$ & $\begin{array}{c}\text { Incoming droplet speed } U \\
\left(\mu \mathrm{m} \mathrm{s}^{-1}\right)\end{array}$ & $\begin{array}{c}\gamma \\
\left(\mathrm{mN} \mathrm{m}^{-1}\right)\end{array}$ & $\begin{array}{c}\text { Capillary number } \\
\mathrm{Ca}\end{array}$ \\
\hline $\begin{array}{l}\text { Fluorinated oil droplets } \\
\text { in DI water+SDS }\end{array}$ & 1.67 & $5.8<U<300$ & 15.4 & $3 \times 10^{-4}<\mathrm{Ca}<1.8 \times 10^{-2}$ \\
$\begin{array}{l}\text { Water droplets } \\
\text { in hexadecane+SPAN80 }\end{array}$ & 0.11 & $2.4<U<165$ & 5 & $3.8 \times 10^{-3}<\mathrm{Ca}<0.27$ \\
\hline \hline
\end{tabular}




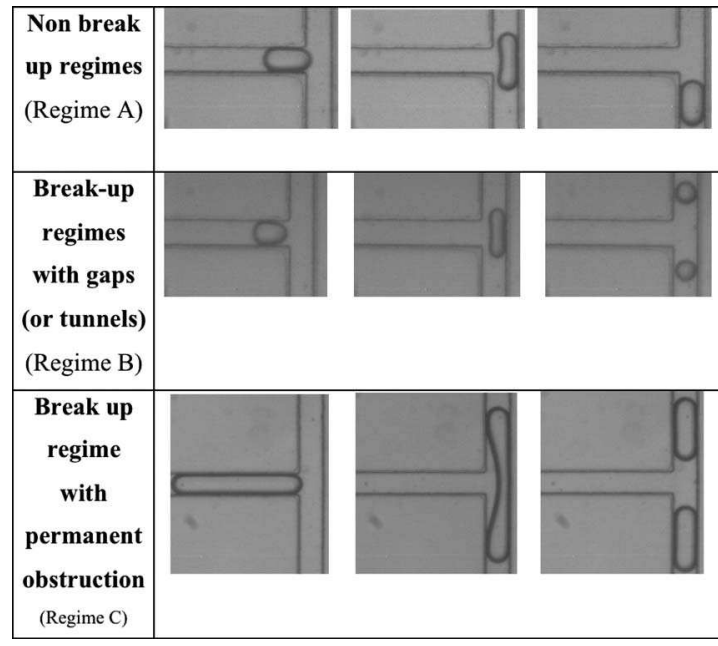

FIG. 2. Three regimes observed in the experiment using fluorinated oil and water $(\lambda=1.67)$; the droplets move from left to right along the horizontal channel. The scales are the same for all the figures, with channel widths equal to $80 \mu \mathrm{m}$. The separation times vary from one regime to the other; they have been determined so as to reveal the major events occurring in each regime. (a) No breakup regimes; (b) breakup with tunnels; (c) breakup with permanent obstruction.

typically for moderate extensions, droplets never obstruct the branches, leaving a tunnel permanently open between the sidewalls and the droplet. Viscous stresses apparently work at elongating the droplet. After the droplet is sufficiently elongated, breakup occurs, generating two daughter droplets. In another case, typically for longer extensions, the droplet penetrates into the junction keeping the two arms (temporarily) obstructed. In this process a neck forms in the central part of the junction with a width decreasing in time. After some time, a tunnel opens that favors the development of a flow of the external phase between the droplet and the sidewall. This flow presumably builds up tangential stresses that work at elongating the droplet. At some point breakup occurs.

(c) Breakup with permanent obstruction [Fig. 2(c)]. The droplet arrives at the junction and penetrates into the two branches of the junction, keeping the channels obstructed. In this process a neck with a circular shape forms in the central part of the junction. This neck thins out with time, until breakup occurs and two daughter droplets form. These droplets further move downstream along the two branches of the T-junction. Throughout this process, the channels keep being obstructed by the droplets.

We now discuss in more detail the difference between obstructed and nonobstructed regimes. We measured the tunnel width $d$ just before breakup occurs as a function of the capillary number in regimes B and C. The result is shown in Fig. 3. In regime $\mathrm{B}$, we find that $d$ increases with the capillary number in a way independent of the droplet extension $l_{0}$. By fitting the data with a power law, we obtain the following expression:

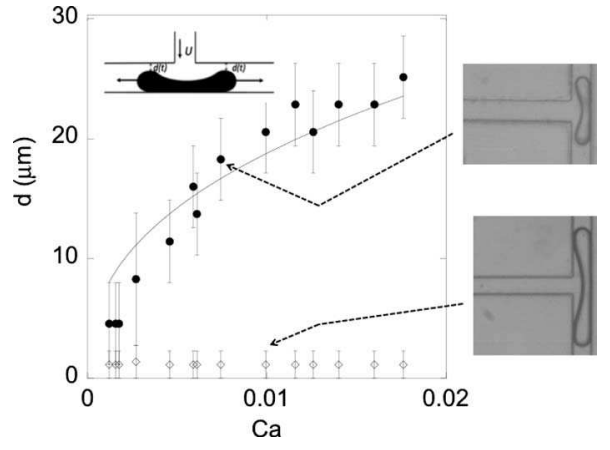

FIG. 3. Minimal tunnel width $d$ preceding breakup onset as a function of capillary number in two cases: $(\bullet)$ regime B (breakup with tunnels); $(\diamond)$ regime $\mathrm{C}$ (breakup with obstruction). The full line represents the expression $1.5 w \mathrm{Ca}^{2 / 5}$. We also present experimental pictures showing the conditions in which $d$ reaches its minimal value.

$$
d=(1.5 \pm 0.1) \mathrm{Ca}^{0.4 \pm 0.05} w,
$$

where $w$ is the channel width.

This law establishes a formal link between our work and the analytical theory of Ref. 10 which found that in two dimensions, $d$ should increase as $\approx w \mathrm{Ca}^{2 / 5}$, in the limit $d \ll w$ (which is approximately the case in our experiments). The power and the prefactor of the power law we found are remarkably close to the theory. By contrast, in regime $\mathrm{C}$, i.e., when the channel is permanently obstructed, $d$ measurements lie below our optical resolution. We obtain in this case a plateau close to the horizontal axis. Figure 3 thus reveals two different evolutions for the tunnel width $d$ as a function of the capillary number, depending whether the droplet obstructs or not the T-junction in the sense we defined above. This observation justifies that beyond geometrical or topological considerations, a distinction must be made between these two cases.

We now study the dynamical behavior of droplets in regime $\mathrm{C}$ (obstructed flows) when they arrive at the T-junction. Specifically, we measured the four geometrical quantities sketched on Fig. 4: the radius of curvature of the rear front $R(t)$, the point where the rear interface joins the upper wall of the right $\mathrm{T}$ arm $x^{\prime}(t)$, its equivalent for the forefront interface $x(t)$, and the neck width $\delta(t)$.

We start by discussing the evolution of $R(t)$, a quantity represented on Fig. 5(a) for different capillary numbers, different droplet lengths, and the same viscosity ratio $\lambda$. The

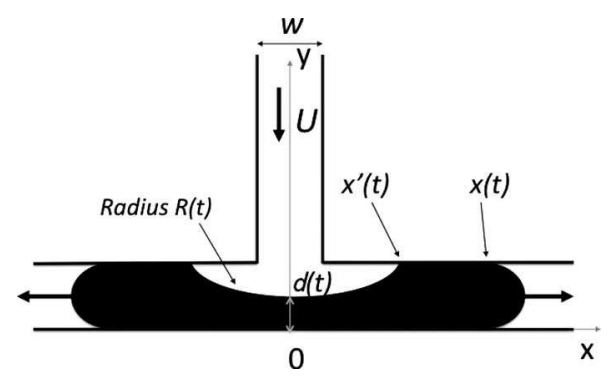

FIG. 4. Sketch of the shape of a droplet arriving at the T-junction and definitions of the four quantities we use in the present study to characterize the corresponding droplet morphologies: $R(t), x^{\prime}(t), x(t)$, and $\delta(t)$. 

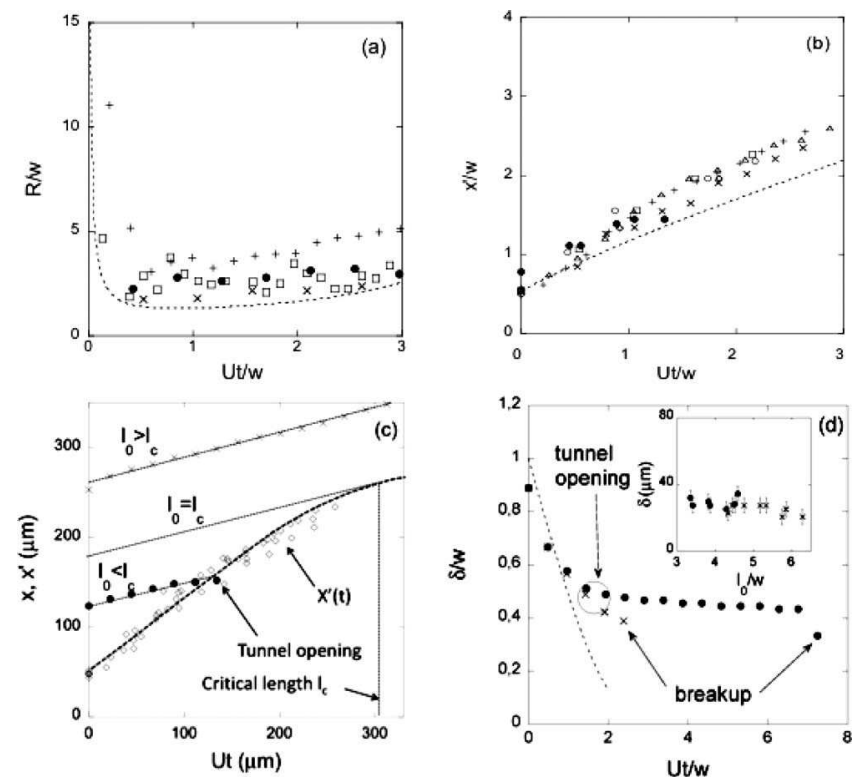

FIG. 5. (a) Radius of curvature over channel width $R / w$ as a function of rescaled time $U t$ (with $U$ as the incoming droplet speed) for several droplet lengths $l_{0}$, different capillary numbers $\mathrm{Ca}$, and for viscosity ratio $\lambda=1.67$. (口) $l_{0} / w=3.77$ and $\log (\mathrm{Ca})=-3.3 ;(\times) l_{0} / w=6$ and $\log (\mathrm{Ca})=-2.5 ;$ $l_{0} / w=4.08, \log (\mathrm{Ca})=-2.8 ;+l_{0} / w=7.54, \log (\mathrm{Ca})=-3.4$. (b) Evolution of $x^{\prime} / w$ as a function of $U t / w$ for different capillary numbers, droplet lengths and viscosity ratios. $(\bigcirc) l_{0} / w=4.96, \log (\mathrm{Ca})=-2.86$, and $\lambda=1.67 ;(\square)$ $l_{0} / w=4.87, \log (\mathrm{Ca})=-2.78$, and $\lambda=1.67 ;(\diamond) l_{0} / w=4.77, \log (\mathrm{Ca})=-2.55$, and $\lambda=1.67 ;(\times) l_{0} / w=6.5, \log (\mathrm{Ca})=-3.21$, and $\lambda=1.67 ;+l_{0} / w=7.66$, $\log (\mathrm{Ca})=-3.1, \lambda=1.67 ;(\triangle) l_{0} / w=7.44, \log (\mathrm{Ca})=-3.04$, and $\lambda=1.67 ;(\bullet)$ $\lambda=0.11$. (c) Evolution of $x(t)$ and $x^{\prime}(t)$ for two droplets with same capillary number $\log (\mathrm{Ca})=-3.12$ and same viscosity ratio $\lambda=1.67$, and different droplet lengths showing the conditions under which a tunnel opens; $x(t)$ data with $(\bullet) l_{0} / w=4.02 ;(\times) l_{0} / w=7.1 ;(\diamond) x^{\prime}(t)$ data. The dashed line represents the universal behavior of $x^{\prime}(t)$ at different capillary numbers. The dotted lines are either fits of the data or the tangent to $x^{\prime}(t)$. (d) Temporal evolution of the neck width $\delta$ for two different droplet lengths and for $\log (\mathrm{Ca})=-2.7,(\times) l_{0} / w=6.28 ;(\bullet) l_{0} / w=3.45$. The inset of (d) represents $d$ measurements at breakup onset as function of droplet extension. On (a)-(c), the dashed lines correspond to the $2 \mathrm{D}$ potential flow simulation that assimilates the interface to a material line.

origin of time is taken when the rear interface reaches the level $y=w$; at this time, the rear interface looks flat. From Fig. 5(a), we observe that $R(t)$ decreases rapidly down to $3-4 w$ and then raises up gradually up to $4-5 w$ over a time scale, measured in unit of $w / U$, on the order of 3. Interestingly, the evolution of $R(t)$ —with time expressed in units of $w / U$-appears to be weakly sensitive to capillary number changes: Indeed, in Fig. 5(a), the capillary number has been varied by more than one order of magnitude while the curves appear, within $30 \%$, to be indistinguishable from each other. The absence of substantial capillary number dependence is observed more strikingly with $x^{\prime}(t)$ : In Fig. 5(b), it appears that $x^{\prime}(t)$-with time expressed in units of $w / U$-does not depend on the capillary number nor on the viscosity ratio $\lambda$.

Here we suggest an explanation for the absence of dependence on the capillary number and the viscosity ratio of $x^{\prime}(t)$ and $R(t)$ along with their functional shape. One may notice that when the rear interface of the droplet penetrates into the T-junction, it develops large radii of curvature (see Fig. 3) and thus small capillary forces. As long as the rear interface appears isolated from the rest of the droplet (i.e., before a tunnel opens), one may consider that it behaves as a simple material line passively advected by the flow pattern that would prevail in the T-junction in the absence of droplet. In order to check whether such a view can be advocated, we performed calculations of the advection of material lines by potential velocity fields in a $2 \mathrm{D}$ T-junction geometry similar to the experiment. The 2D potential approximation we used would be accurate with shallow microchannels (Hele-Shaw type flows); we assume here that it provides qualitatively correct results for our square cross-sectional microchannels, for which the degrees of freedom in the vertical dimension appear frozen. The calculations were performed with MATLAB software. The evolutions of $x^{\prime}(t)$ and $R(t)$ that we obtained numerically are represented in Figs. 5(a), 5(b), and $5(d)$ as dashed lines. Within $30 \%$, the theoretical profile of $x^{\prime}(t)$ is found to represent the observed evolutions of these quantities. Concerning $R(t)$, the agreement is more qualitative. As a whole, the calculation supports the idea that we can roughly assimilate the rear droplet interface as a material line. This argument-particularly counterintuitive for the low capillary numbers we explore here-provides a hint for understanding why the evolutions of $R(t)$ and $x^{\prime}(t)$ are found to be independent of the capillary number and the viscosity ratio.

We now turn to the discussion of the evolution of $x(t)$ shown in Fig. 5(c) for a viscosity ratio $\lambda$ equal to 1.67 and a capillary number equal to $7.5 \times 10^{-4}$ and two distinct values of $l_{0}\left(l_{0} / w=4.02\right.$ and $\left.l_{0} / w=7.1\right)$. We find that $x(t)$ increases linearly with time. The law for the temporal evolution of $x(t)$ can be expressed in the following form:

$$
x(t) \approx \beta \frac{U}{2} t+\frac{l_{0}-w}{2},
$$

with $\beta \sim 0.7$. Should the obstruction be complete and the flow rate be imposed, we would have found $\beta=1$. The fact that $\beta$ is smaller than unity remains to be fully explained. One possibility is that as the droplet arrives in the T-junction, the droplet morphology changes and favors, owing to the action of capillarity, an increase in the pressure at the rear of the droplet. Since we operate at fixed pressure, this should provoke a general reduction of the flow rate. This mechanism may explain why $\beta$ is smaller than unity. Note that a pressure increase at the rear of the droplet was observed numerically for droplet generators in T-junctions. ${ }^{8}$

From the analysis of the evolutions of $x(t)$ and $x^{\prime}(t)$, we can argue that the conditions of tunnel formation are controlled by a critical length, as we announced in the abstract of the paper. The discussion is substantiated in Fig. 5(c). Plotted as a function of $U t$, the line that represents $x(t)$ has a slope equal to $\beta$ and a position that depends on the droplet extension $l_{0}$. This line is independent of the capillary number. On the other hand, $x^{\prime}(t)$ plotted as a function of $U t$ is a "universal" curve. Thus, whether $x(t)$ and $x^{\prime}(t)$ intersect (i.e., whether a tunnel opens or not) is entirely based on the droplet extension $l_{0}$ and $\lambda$. For moderately long droplets, the two curves cross and consequently a tunnel opens. For much longer droplets, the curve $x(t)$ lies above $x^{\prime}(t)$ and no intersection is possible; consequently no tunnel forms in this 

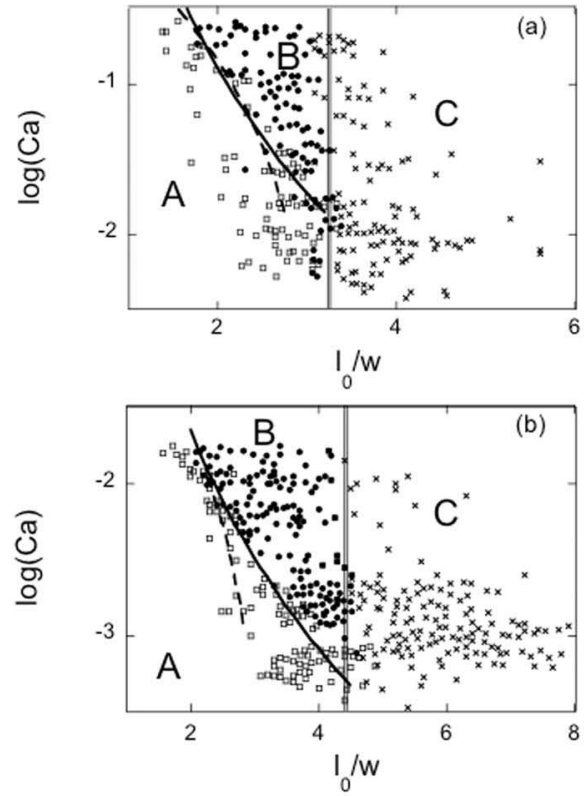

FIG. 6. Phase diagrams representing the behaviors of isolated droplets in T-junctions for two different viscosity ratios of the phases in presence: (a) $\lambda=0.11$; (b) $\lambda=1.67$. The various regimes are (A) no breakup, (B) breakup with tunnels, and $(\mathrm{C})$ obstructed breakup. The double vertical line represents the critical length $l_{C} / w$ over which no tunnel forms, the full line represents the theory of Ref. 10, and the dashed curve represents the theory of Ref. 5.

case. In between the two cases, a critical droplet length $l_{c}$ can be defined. This critical length is the length for which $x(t)$ is tangent to $x^{\prime}(t)$ [this is shown in Fig. 5(c)]. Following the above definition, the critical length can be estimated to be $l_{C} \sim 340 \pm 50 \mu \mathrm{m}$ for $\lambda=1.67$. A similar construction for $\lambda=0.11$ leads to $260 \pm 30 \mu \mathrm{m}$ for $l_{C}$.

We now comment on the behavior of the neck as the droplet moves through the T-junction. Figure 5(d) represents the evolutions of $\delta(t)$ for droplets belonging to regimes B and $\mathrm{C}$. In regime $\mathrm{B}$, the width of the neck decreases rapidly with time up to a point where the tunnel opens; after this event, the neck still thins out but at a smaller rate. In all cases, droplets eventually breakup when the neck reaches a critical width $\delta_{C}$ equal to (see the inset),

$$
\delta_{C} \approx 0.3 w \text {. }
$$

The existence of a critical width was proposed in Ref. 10 and the value we obtained here is remarkably close to the $2 \mathrm{D}$ estimate $\delta_{C} \approx w / 2$. Our observation suggests in fact that $2 \mathrm{D}$ theory can be used to interpret the experiment. In regime C, the neck thins out at a fast rate (roughly given by the 2D simulation) up to a point where breakup occurs. Note that there seems to exist also a critical neck width in regime $\mathrm{C}$.

We now represent the regimes $\mathrm{A}-\mathrm{C}$ on a capillaryextension diagram for the two liquid systems we investigated [see Figs. 6(a) and 6(b)]. Each regime is identified by a particular symbol. It is worthwhile noting that however different, the phase diagrams of the two liquid systems have similar structures. The diagram can be used to compare the theories of Ref. 5 to the experiment in an unprecedented range of capillary numbers. The theoretical prediction of Ref. 5 is given by

$$
\mathrm{Ca}=\alpha \frac{l_{0}}{\pi w}\left[\left(\frac{\pi w}{l_{0}}\right)^{2 / 3}-1\right]^{2},
$$

in which $\alpha$ is an adjustable parameter. The best values we found are $\alpha=1.8$ for $\lambda=0.11$ and $\alpha=0.25$ for $\lambda=1.67$. These values are consistent with Refs. 5 and 9. Formula (2) describes the boundaries between regimes A and B in the upper range of the capillary numbers but fails to describe the breakup conditions we observed at smaller capillary numbers. In the theory of Leshansky and Pismen ${ }^{10}$ the boundary between regions $\mathrm{A}$ and $\mathrm{B}$ is given by the following formula:

$$
l_{0} / w \approx \chi \mathrm{Ca}^{-0.21},
$$

with $\chi \approx 0.98$ in the $2 \mathrm{D}$ case. ${ }^{11}$ We found that our measurements are compatible with the above formula using $l_{0} / w$ with $\chi \approx 1.3$ for $\lambda=0.11$ and $\chi=0.9$ for $\lambda=1.67$. As a whole, the formula of Leshansky and Pismen agrees well with the experiment, within a range of capillary numbers extending over more than 1 decade.

We now discuss regime $\mathrm{C}$ in which droplets obstruct the T-junction and eventually breakup. In Figs. 6(a) and 6(b), the boundaries of region $\mathrm{C}$ are well represented by doubled vertical lines associated to critical lengths. Beyond such "critical lines," no tunnel forms and breakup is unavoidable. Region $\mathrm{C}$ is in fact defined by a geometrical rather than a dynamical condition and this condition finds a particularly simple expression with the notion of critical length we introduced earlier.

To conclude, we have extended the description of droplet breakup at T-junctions, encompassing a broader range of capillary numbers than explored thus far in literature. We revealed two regimes depending whether droplets obstruct or not the T-junction: For droplets that do not obstruct the junction, our observations agree with the theory of Leshansky and Pismen ${ }^{10}$ at a semiquantitative level. More precisely, we observed the following features:

- a dynamical behavior of the droplet at the junction corresponding well to the theoretical framework;

- an evolution of the minimal gap width as $1.5 w \mathrm{Ca}^{0.4 \pm 0.05}$ law (to be compared with the $2 \mathrm{D}$ estimate $1.0 w \mathrm{Ca}^{2 / 5}$ );

- a neck size at breakup equals to 0.3 times the channel width at all capillary numbers (to be compared with 0.5 times the channel width in the theory);

- breakup conditions consistent with the predicted $\mathrm{Ca}^{-0.21}$ law with a prefactor comparable to the theory.

Although the theory was conducted in two dimensions, it is remarkable that it describes so accurately our (threedimensional) experiment. The fact that in the experiment, degrees of freedom are frozen in the vertical dimension may perhaps explain this success.

The theory of Ref. 10 does not however describe all the regimes we observed: For the theory to apply, the droplet must develop a tunnel. This excludes the regimes we called "permanently obstructed" in which there is no observable gap between the droplet and the wall. Using physical arguments, we described such regimes by introducing a critical droplet length that is independent on the capillary number. 
The idea of introducing a characteristic length to describe the breakup process in microfluidic junctions is not new; it was proposed for asymmetric junctions in Ref. 6. The extent to which such notion can be generalized is nonetheless unclear.

The above remarks confirm that a classification between obstructed and nonobstructed regimes must be done to describe breakup in T-junctions. Note that the idea of distinguishing obstructed and nonobstructed regimes imposes itself in the droplet generation problem. ${ }^{8}$ In this paper, different breakup regimes exist, depending on whether the droplet generated at the junction blocks the junction (squeezing regime) or not (dripping regime). Whether droplet obstructs or not, the channels must probably be taken seriously, in general, in digital microfluidics.

We would like to thank A. Leshansky, L. Pismen, H. Stone and his group, D. Weitz, P. Garstecki, A. Ajdari, Y. Hennequin, P. Nghe, H. Willaime, and N. Pannacci for fruitful discussions related to this work. Annick Marin is warmly acknowledged for her help. CNRS, Ecole Supérieure de Physique et Chimie de Paris and MIT are gratefully acknowledged for their supports.
${ }^{1}$ B. Zheng and R. F. Ismagilov, "A microfluidic approach for screening submicroliter volumes against multiple reagents by using preformed arrays of nanoliter plugs in a three-phase liquid/liquid/gas flow," Angew. Chem., Int. Ed. 44, 2520 (2005).

${ }^{2} \mathrm{H}$. Song and R. F. Ismagilov, "Millisecond kinetics on a microfluidic chip in multiphase microfluidics at low values of the Reynolds and capillary numbers," J. Am. Chem. Soc. 125, 14613 (2003).

${ }^{3} \mathrm{~K}$. Jensen and A. Lee, "The science and applications of droplets in microfluidics devices," Lab Chip 4, 31N (2004).

${ }^{4} \mathrm{P}$. Tabeling, Introduction to Microfluidics (Oxford University Press, Oxford, 2005).

${ }^{5}$ D. R. Link, S. L. Anna, D. A. Weitz, and H. A. Stone, "Geometrically mediated breakup of drops in microfluidic devices," Phys. Rev. Lett. 92, 054503 (2004).

${ }^{6} \mathrm{~L}$. Ménétrier-Deremble and P. Tabeling, "Droplet breakup in microfluidic junctions of arbitrary angles," Phys. Rev. E 74, 035303 (2006).

${ }^{7}$ P. Garstecki, M. Feuerstman, H. A. Stone, and G. M. Whitesides, "Formation of droplets and bubbles in a microfluidic T-junction: Scaling and mechanism of breakup," Lab Chip 6, 437 (2006).

${ }^{8}$ M. De Menech, P. Garstecki, F. Jousse, and H. A. Stone, "Transition from squeezing to dripping in a microfluidic T-shaped junction," J. Fluid Mech. 595, 141 (2008).

${ }^{9} \mathrm{M}$. De Menech, "Modeling of droplet breakup in a microfluidic T-shaped junction with a phase-field model," Phys. Rev. E 73, 031505 (2006).

${ }^{10}$ A. M. Leshansky and L. M. Pismen, "Breakup of drops in a microfluidic T junction," Phys. Fluids 21, 023303 (2009).

${ }^{11}$ In Ref. 10 the authors refer to the maximal extension and found $\chi=1.3$. In a private communication the authors communicated to us the value related to the initial extension. 